\title{
Incentives in Academics: Collaboration under Weak
}

\section{Complementarities}

March 31, 2005

Junichiro Ishida

Associate Professor, Osaka School of International Public Policy (OSIPP)

【キーワード】 Collaboration; Complementarities; Incentives; Contracts; Peer sanction. 【要約】 This paper considers a contracting relationship with multiple agents in a repeated setting under voluntary team formation. In each period, an opportunity to collaborate arrives stochastically but whether this opportunity has arrived is the agents' private information. The principal thus cannot simply tell them when to collaborate; she must instead guide them through incentives. In this situation, there may arise an incentive for the principal to encourage collaboration in the repeated setting as it allows her to exploit the agents' ability to monitor each other. We then argue that low-powered incentives offered for non-collaborative works, as often observed in our profession, can be seen as an important part of optimal incentive schemes in an attempt to endogenously raise the cost of shirking. We also show that this type of compensation scheme is socially inefficient and detrimental to the agents when the discount factor is near unity.

Financial support from the Japanese Ministry of Education, Culture, Sports, Science and Technology (Grants-in-Aid for Young Scientists (B), 14730013, 2002) is gratefully acknowledged. Correspondence to: Junichiro Ishida, OSIPP, Osaka University, 1-31 Machikaneyama, Toyonaka, Osaka 560-0043, Japan. E-mail: jishida@osipp.osaka-u.ac.jp 


\section{Introduction}

Collaboration is a crucial aspect of academic research in many fields. This tendency is certainly more prevalent in natural sciences, where a bulk of research projects are conducted by multiple researches. The situation is, however, not far different in social sciences and especially in economics. A recent tendency is that the number of collaborative works in economics increases rapidly and now occupies a substantial portion of all published papers. ${ }^{1}$

There are presumably many reasons why researchers collaborate. From their viewpoint, though, it ultimately comes down to how the amount of output is related to pecuniary (and sometimes nonpecuniary) compensations such as job offers, tenures, grants, promotions and raises in base salary. To illustrate this point, consider the world of no complementarity where writing one single-authored paper requires exactly the same amount of energy as writing two coauthored papers (when collaborated by two researchers). In this world, a 'fair' way to compensate is to count one single-authored paper as equivalent to two coauthored papers. If two coauthored papers do count more heavily for some reasons, self-interested researchers actually have an incentive to collaborate, just for their own sake. Although it is difficult to verify this claim empirically, we take it rather uncontroversial that there is a tendency to reward lengthy curriculum vitae in academics (or at least in economics), implying that two coauthored papers indeed do count more heavily than one single-authored paper of identical quality. $^{2}$ Alternatively, this implies that researchers are given relatively low-powered incentives for non-collaborative works conducted individually.

Given this, the question we need to ask at this juncture is why the compensation scheme in our profession is designed in such a way that seems to encourage collaboration. An obvious reason for this is perhaps the presence of complementarities: the contract designer (the principal) encourages collaboration to reap the benefit of complementarities. Researchers can most likely benefit from

\footnotetext{
${ }^{1}$ See, for instance, Heck and Zaleski (1991), Hudson (1996) and Engers (1999) et al. Also, see Laband and Tollison (2000) for the difference in the trend of collaboration between economics and biology.

${ }^{2}$ Due to the nature of the issue, empirical evidence on this is relatively sparse and hardly conclusive: for instance, Sauer (1988) fails to reject that $n$ coauthored papers (with $n$ authors) counts more heavily than a single-authored paper in determining salaries of academic ecnomists, using cross-section data. It is documented in survey data, however, that universities do not typically fully discount collaborative work by the number of authors (McDowell and Smith, 1992).
} 
complementarities when a task requires various specialized skills. In this case, collaboration is valuable as it realizes the efficient division of labor by allowing each participant to focus on narrowly defined tasks. A benefit of collaboration may also arise even between researchers with a similar set of skills because collaboration may lead to an idea none could have come up with individually. ${ }^{3}$ This effect is more important when two or more specialists in the same fields work jointly, as we often observe in economics. When the effect of complementarities is present, there arise potential benefits for the contract designer to encourage collaboration since it leads to more output, given the same amount of effort.

While this reasoning sounds fairly convincing, collaboration also poses a cumbersome problem when each researcher's effort is not directly observable, as is normally the case. Under collaboration, observable signals of effort are more likely to become less sensitive to each researcher's effort now that they are also dependent on the collaborator's effort as well. The cost of inducing effort from each researcher naturally increases since each can free-ride on the collaborator's effort. To justify the compensation scheme which seems to encourage collaboration, the degree of complementarities in the production process needs to be strong enough to compensate for the loss arising from the free-rider problem: in fact, under plausible circumstances, the degree of complementarities needed to outweigh this free-rider problem is fairly substantial. We argue that this is doubtful, especially in economics where skill complementarities do not appear that critical. ${ }^{4}$ Indeed, empirical evidence on the effect of collaboration on academic output in economics is mixed at best. ${ }^{5}$

Provided that the presence of complementarities alone does not necessarily justify the current compensation scheme, we focus on another potential source of benefit, that is, each researcher's desire to maintain reputation in the academic community. The critical aspect of the model is that collaboration allows researchers to work closely with each other and thus to observe what the contract

\footnotetext{
${ }^{3}$ This effect is often referred to as synergies to distinguish them from skill complementarities. In the paper, however, we do not make any particular distinction between them.

${ }^{4}$ This may be in sharp contrast to natural sciences where the division of labor is clearer, compared to social sciences.

${ }^{5}$ McDowell and Smith (1992) finds no significant evidence that collaboration enhances productivity while Durden and Perri (1995) finds evidence that collaboration indeed has a positive effect. Recently, using panel data, Hollis (2001) shows that the effect of collaboration on output is even negative after discounting for the number of authors.
} 
designer cannot observe, namely each researcher's effort level. Since no one would like to collaborate with lazy and dishonest colleagues, each researcher has an additional motivation, absent when working individually, to present himself as hard-working and honest. This motivation becomes stronger when collaboration is so much more attractive for researchers: in this case, it can simply be too costly to shirk and destroy a cooperative relationship with able colleagues. The contract designer can then exploit this incentive to maintain reputation by offering low-powered incentives for non-collaborative work in order to endogenously raise the cost of shirking.

To formalize this situation we consider a contracting situation in an infinitely repeated setting under voluntary team formation. There is now a growing body of literature on so-called relational contracts, which is an attempt to explicitly incorporate repeated interactions into the contract theory. ${ }^{6}$ The idea that the agents' ability to monitor each other can be exploited by appropriately designing compensation schemes is first pointed out and formulated by Che and Yoo (2001). ${ }^{7}$ This paper builds on this framework and extends it to a situation under voluntary team formation. The situation we will analyze in this paper is as follows. Consider a situation where a principal hires two agents and lets them perform a task either individually or jointly. In each period, an opportunity to collaborate arrives stochastically, but whether this opportunity has arrived is the agents' private information. Not knowing when collaboration is feasible, the principal cannot simply tell them when to collaborate; she must instead provide incentives to collaborate when she wants them to by appropriately designing the compensation scheme.

In this framework there arises a link between incentives for collaborative works and those for non-collaborative works. Consider a trigger strategy where an agent's defection leads to perpetual retaliation by the other agent. The cost of defection is certainly small if each agent can earn sufficiently large rents from non-collaborative works because he can always work individually. The principal then has an incentive to offer low-powered incentives for non-collaborative works so that the agents cannot earn sufficient rents from it. We identify conditions under which it is indeed optimal to offer this

\footnotetext{
${ }^{6}$ To name a few, Bull (1987), MacLeod and Malcomson (1989), Baker, Gibbons and Murphy (1994, 2002), Bernheim and Whinston (1998), MacLeod (2003) and Levin (2003) explore the optimal form of contract in long-term relationships.

${ }^{7}$ Also, see Rayo (2003) and Ishida (2004) on team incentives in long-term relationships.
} 
type of contract under arbitrarily weak complementarities. We also show that this strategy is socially inefficient and especially detrimental to the agents when the discount factor is near unity. This implies that our desire to maintain reputation in the academic community may backfire and actually hurt us when the compensation scheme is designed to take advantage of it.

The rest of the paper is organized as follows. The next section outlines the basic environment. Section 3 analyzes the static version of the model as a benchmark. Section 4 extends the analysis to the repeated setting and show how the optimal compensation scheme from that in the static setting and its effect on social welfare. Finally, section 5 offers some concluding remarks.

\section{Model}

\subsection{Environment}

Consider a model with a principal (female) and two agents (male, $i=A, B$ ) in an infinitely repeated setting. Time is discrete and denoted by $t \in T \equiv\{0,1,2, \ldots\}$. All parties are assumed to be risk neutral. The common discount factor is given by $\delta \in(0,1)$.

In each period, the principle lets $A$ and $B$ engage in a task. Each task is characterized by the number of agents to work on the task, which we refer to as the mode of production. The mode of production is denoted by superscript $j \in\{c, n\}$ where $j=c$ if the agents work jointly ( $c$ for collaboration) and $j=n$ if they work individually ( $n$ for non-collaboration).

An opportunity to collaborate arrives stochastically. With probability $\theta \in(0,1)$, the opportunity arrives and the agents must decide whether to take this opportunity and work jointly. ${ }^{8}$ The agents can work jointly only if both of them agree to do so. The agents work individually if either (i) the opportunity does not arrive or (ii) they fail to reach an agreement. Whether this opportunity has arrived is the agents' private information and cannot be observed by the principal.

\footnotetext{
${ }^{8}$ There are many potential interpretations for the parameter $\theta$. One is to regard this as the probability of finding a suitable collaborator. It is also possible to interpret this as the probability that the agents successfully come up with a suitable research idea on which both of them can agree to collaborate.
} 


\subsection{Production}

In each period, each agent must exert costly effort to produce output. The effort choice is binary and denoted by $e_{i, t} \in\{0,1\}$. The cost of effort is given by $d e_{i, t}$ for non-collaborative work and $\gamma d e_{i, t}$, $\gamma \in(0,1)$, for collaborative work. The parameter $\gamma$ measures the degree of complementarities in the production process, and its effect disappears as $\gamma \rightarrow 1$. The effort choice is not observable to the principal.

The task is either successful or not, and its outcome is denoted by $y_{i, t} \in\{0,1\}$. For practical purposes, $y_{i, t}$ can be interpreted as the number of publications per author in a given period. ${ }^{9}$ When the agents work individually, the probability of a success is given by

$$
\operatorname{prob}\left(y_{i, t}=1 \mid e_{i, t}\right)=p_{1} e_{i, t}+p_{0}\left(1-e_{i, t}\right), p_{1}>p_{0}>0 .
$$

The output of agent $i$ thus depends solely on his own effort level. Define $\Delta p \equiv p_{1}-p_{0}$.

When the agents work jointly, on the other hand, the probability of a success is given by

$$
\operatorname{prob}\left(y_{i, t}=y_{-i, t}=1 \mid e_{i, t}, e_{-i, t}\right) \equiv f\left(e_{i, t}, e_{-i, t}\right), i \neq-i .
$$

For the analysis we consider a simple additive technology to emphasize that the only source of complementarities is the difference in the cost of effort: $f(1,1)=p_{1}, f(0,0)=p_{0}$ and $f(1,0)=f(0,1)=$ $0.5\left(p_{0}+p_{1}\right)$. Under this specification, an agent's marginal value of effort is independent of the other agent's effort level: that is,

$$
f(1,1)-f(0,1)=f(1,0)-f(0,0)=0.5 \Delta p .
$$

In each period, the principal earns a private benefit $V\left(y_{A, t}+y_{B, t}\right)$, depending on the outcome. Notice that the principal simply values the number of publications per author so that there is no inherent need to reward collaboration more heavily in this sense.

\footnotetext{
${ }^{9}$ That is, $y_{i, t}=1$ means either one single-authored publication (if agent $i$ works individually) or two coauthored publications (if he collaborates).
} 


\subsection{Contract}

The principal makes a take-it-or-leave-it offer to the agents in order to maximize the expected profit. ${ }^{10}$ The reservation payoff for both parties is assumed to be zero. Throughout the analysis, we focus on a case where the contract offered initially applies for all subsequent periods. The principal can observe the mode of production and the outcome of the task. There are thus four possible cases, and the contract offered by the principal specifies the wage for each contingency. Let $\mathbf{w}^{j}=\left(w_{0}^{j}, w_{1}^{j}\right), j=c, n$, denote a contract where $w_{y}^{j} \in[0, \infty)$ is the wage when the mode of production is $j \in\{c, n\}$ and the outcome is $y \in\{0,1\}$ : for expositional purposes we sometimes refer to $\mathbf{w}^{n}$ as the individual contract and to $\mathbf{w}^{c}$ as the team contract. Also, let $\mathbf{w}=\left(w_{0}^{n}, w_{1}^{n}, w_{0}^{c}, w_{1}^{c}\right)$. Finally, there is a liquidity constraint such that the principal must offer nonnegative wages.

Before we proceed further, we need to make an assumption concerning the possibility of 'fake collaboration.' Since the principal is unable to observe when collaboration is feasible, there is a possibility that the agents claim to have engaged in collaboration even though they in fact worked individually. This possibility may severely limit the effectiveness of a contract which rewards collaboration more heavily. While it is conceptually possible, however, this behavior is a clear violation of a professional code and we hence rule out this possibility at the outset. There are indeed several reasons why the agents do not falsely pretend to collaborate. First, if there is some (possibly very small) probability that fake collaboration is detected by the principal, and the potential penalty for such a violation (in a broad sense) is very large, it is not beneficial to falsely pretend to collaborate. Second, there may arise some psychological cost (or guilt) associated with being credited in a work that s/he has nothing to do with. In any event, we do not believe that this (fake collaboration) is a serious problem in the academic community, and we thus take the stance that there must be some mechanisms which prevent researchers from falsely pretending to collaborate.

\footnotetext{
${ }^{10}$ In other words, we assume that the principal possesses full bargaining power.
} 


\section{Static benchmark}

We first consider a static version of the model as a benchmark. When the agents work individually, each agent exerts effort if

$$
p_{1} w_{1}^{n}+\left(1-p_{1}\right) w_{0}^{n}-d \geq p_{0} w_{1}^{n}+\left(1-p_{0}\right) w_{0}^{n} .
$$

The incentive compatibility constraint for non-collaborative work is then given by

$$
\Delta p\left(w_{1}^{n}-w_{0}^{n}\right) \geq d
$$

For expositional purposes, we say that a contract is high-powered if it induces effort from each agent; on the other hand, we say that a contract is low-powered if it does not.

Now suppose that the agents choose to engage in collaboration. Taking the other agent's effort level $e_{-i, t}$ as given, each agent exerts effort if

$$
f\left(1, e_{-i, t}\right) w_{1}^{c}+\left(1-f\left(1, e_{-i, t}\right)\right) w_{0}^{c}-\gamma d \geq f\left(0, e_{-i, t}\right) w_{1}^{c}+\left(1-f\left(0, e_{-i, t}\right)\right) w_{0}^{c}
$$

The incentive compatibility constraint for collaborative work is then given by

$$
\left(f\left(1, e_{-i, t}\right)-f\left(0, e_{-i, t}\right)\right)\left(w_{1}^{c}-w_{0}^{c}\right)=0.5 \Delta p\left(w_{1}^{c}-w_{1}^{c}\right) \geq \gamma d .
$$

Given these, each agent must decide whether to collaborate with the other agent when the opportunity arrives. Let $u^{j}$ denote the expected stage-game payoff for each agent, conditional on the mode of production. Upon the arrival of the opportunity, the agents agree to collaborate if $u^{c} \geq u^{n}$.

It is exceedingly tedious to completely characterize the optimal form of contract for a full range of parameters without yielding much insight. We thus confine our attention to a subset of parameters by the following assumptions.

Assumption $1 \Delta p V \geq \frac{d}{\Delta p}$.

Assumption $2 \gamma>0.5$. 
Assumption 1 indicates that effort is sufficiently valuable for the principal ( $d$ is sufficiently small relative to $V$ ) so that the optimal contract is always high-powered in the static setting. The assumption also implies that it is efficient to exert effort. Assumption 2 is what we refer to as the condition of weak complementarities, which limits the lower bound for $\gamma$. Under those assumptions we can state the following result (the role of the assumptions will be made clearer in the proof of proposition 1).

Proposition 1 Under Assumptions 1 and 2, the optimal static contract is given by

$$
\mathbf{w}=\left(0, \frac{d}{\Delta p}, 0,0\right)
$$

Proof: See Appendix.

It can be seen from above that it is more costly for the principal to induce effort when the agents work jointly, because an agent can free-ride on the other agent's effort. In other words, the principal must offer more to induce any given level of effort because each agent's payoff is now less sensitive to his behavior when the agents work jointly. The proposition indicates that the principal never encourages collaboration in the static setting unless the production process exhibits sufficiently strong complementarities. The magnitude of complementarities may need to be quite substantial to compensate for the loss arising from the free-riding problem. In the present setup, collaboration is more profitable only when $\gamma<0.5 .^{11}$

\section{Collaboration through repeated interactions}

\subsection{Analysis}

We now extend the analysis to a repeated setting and examine how the principal should modify the compensation scheme to exploit the possibility of mutual monitoring between the agents. In the repeated setting, the agents work closely with each other when they choose to work jointly. As a

\footnotetext{
${ }^{11}$ In the present setup, $\gamma=0.5$ implies that writing four coauthored papers is equivalent to writing one single-authored
} paper in terms of the amount of effort needed. 
consequence, there arises a possibility that the agents sustain mutual cooperation by adopting a type of trigger strategy.

Suppose that collaboration allows each agent $i$ to observe the other agent's effort level $e_{-i, t}$. Let a history in period $t$ be a sequence of observable effort choices (i.e., the other agent's effort level when they collaborate) up to period $t-1$. Then, a strategy profile is a sequence of functions which map from any possible history in each period into a probability distribution over effort choices in that period. ${ }^{12}$ Within this framework, the equilibrium concept we adopt is similar to Che and Yoo (2001): given some contract, the agents select an equilibrium which yields the highest total payoff among all possible subgame perfect equilibria. Now define two states of the economy, $S \in\{C, N\}$ and consider the following trigger strategy. The economy is initially in state $C$ until a defection occurs: in state $C$, the agents always choose to exert effort whenever they work jointly (if the team contract is high-powered). Once a defection occurs, the economy moves into state $N$ where the agents simply play the stage-game Nash equilibrium ever after. Taking this into account, each agent decides whether to work jointly when the opportunity arrives.

First, even in the repeated setting, the incentive compatibility constraint for non-collaborative works is unchanged because his choice of effort has no future implication under the trigger strategy described above. This means that an agent exerts effort if $\Delta p\left(w_{1}^{n}-w_{0}^{n}\right) \geq d$.

The incentive compatibility constraint for collaborative works needs to be modified, on the other hand, because the principal may exploit the agents' ability of mutual monitoring sustained through repeated interactions. Given this set of strategies, the expected payoff for each agent depends also on the state of the economy. Let $u_{S}^{c}$ denote the expected payoff for each agent in state $S$ when the agents choose to work jointly. Further, let $U_{S}, S=C, N$, denote the expected discounted sum of payoffs in state $S$ :

$$
U_{S}=\frac{1}{1-\delta}\left(\theta \max \left\{u^{n}, u_{S}^{c}\right\}+(1-\theta) u^{n}\right) .
$$

\footnotetext{
${ }^{12}$ We thus do not consider strategies which depend on the history of outcomes $y_{i, t}$.
} 
The incentive compatibility constraint for collaborative works in the repeated setting is then given by

$$
f\left(1, e_{-i, t}\right) w_{1}^{c}+\left(1-f\left(1, e_{-i, t}\right)\right) w_{0}^{c}-\gamma d+\delta U_{C} \geq f\left(0, e_{-i, t}\right) w_{1}^{c}+\left(1-f\left(0, e_{-i, t}\right)\right) w_{0}^{c}+\delta U_{N}
$$

With some algebra, this can be simplified to

$$
0.5 \Delta p\left(w_{1}^{c}-w_{0}^{c}\right)+\delta\left(U_{C}-U_{N}\right) \geq \gamma d
$$

where

$$
U_{C}-U_{N}=\frac{\theta}{1-\delta}\left(\max \left\{u^{n}, u_{C}^{c}\right\}-\max \left\{u^{n}, u_{N}^{c}\right\}\right)
$$

The incentive compatibility constraint for collaborative works is now weaker than in the static setting as long as $U_{C}>U_{N}$. Note that the incentive compatibility constraint for collaborative works now includes not only $\mathbf{w}^{c}$ but also $\mathbf{w}^{n}$.

Given this, the agents must decide whether to collaborate when the opportunity arrives. The condition for this is virtually the same as in the static setting, except that it is now dependent on the state of the economy: in state $S$, the agents choose to work jointly if

$$
u_{S}^{c}-u^{n} \geq 0
$$

Finally, when collaboration is preferred by the agents and the team contract is high-powered, the contract must be collusion-proof to implement the desirable outcome as a team equilibrium:

$$
\begin{gathered}
p_{1} w_{1}^{c}+\left(1-p_{1}\right) w_{0}^{c}-\gamma d \geq p_{0} w_{1}^{c}+\left(1-p_{0}\right) w_{0}^{c}, \\
p_{1} w_{1}^{c}+\left(1-p_{1}\right) w_{0}^{c}-\gamma d \geq 0.5\left(p_{0}+p_{1}\right) w_{1}^{c}+\left(1-0.5\left(p_{0}+p_{1}\right)\right) w_{0}^{c}-0.5 \gamma d .
\end{gathered}
$$

These constraints assure that the agents as a team cannot be made better off by deviating from the equilibrium. As it turns out, these constraints are identical and can be simplified to

$$
w_{1}^{c}-w_{0}^{c} \geq \frac{\gamma d}{\Delta p}
$$

We now investigate whether there are circumstances under which it is optimal for the principal to offer a contract which encourages collaboration. There are generically three distinct types of contract 
that the principal can offer in this situation: (i) only the individual contract is high-powered; (ii) both of the contracts are high-powered; (iii) only the team contract is high-powered. ${ }^{13}$ In what follows we explore each case in turn.

\section{Type 1: Only the individual contract is high-powered}

Consider a case where the principal offers a high-powered individual contract combined with a low-powered team contract. This is the case if

$$
\Delta p\left(w_{1}^{n}-w_{0}^{n}\right) \geq d \quad \text { and } \quad \gamma d>0.5 \Delta p\left(w_{1}^{c}-w_{0}^{c}\right)+\delta\left(U_{C}-U_{N}\right)
$$

We denote the expected profit that the principal earns from each agent in this case by $\pi_{h l}$. If $u^{n}>u_{C}^{c}$, the expected profit per agent is given by

$$
\pi_{h l}=p_{1}\left(V-w_{1}^{n}\right)-\left(1-p_{1}\right) w_{0}^{n} .
$$

Note that if the team contract is low-powered, no peer sanction mechanism can function because $u_{C}^{c}=u_{N}^{c}=p_{0} w_{1}^{c}+\left(1-p_{0}\right) w_{0}^{c}$. This implies that $U_{C}=U_{N}$ and the problem is simply reduced to its static version. The optimal contract in this case is exactly as described in proposition 1 . The next result directly follows from proposition 1 (the proof abbreviated).

Lemma 1 Consider a class of contracts in which only the individual contract is high-powered. Under Assumptions 1 and 2, the optimal contract within this class is given by

$$
\mathbf{w}=\left(0, \frac{d}{\Delta p}, 0,0\right)
$$

\section{Type 2: Both of the contracts are high-powered}

We now consider a case where both of the contracts are high-powered, i.e.,

$$
\Delta p\left(w_{1}^{n}-w_{0}^{n}\right) \geq d \quad \text { and } \quad 0.5 \Delta p\left(w_{1}^{c}-w_{0}^{c}\right)+\delta\left(U_{C}-U_{N}\right) \geq \gamma d
$$

We denote the expected profit in this case by $\pi_{h h}$. If $u_{C}^{c} \geq u^{n}$, the expected profit per agent is given by

$$
\pi_{h h}=\theta\left(p_{1}\left(V-w_{1}^{c}\right)-\left(1-p_{1}\right) w_{0}^{c}\right)+(1-\theta)\left(p_{1}\left(V-w_{1}^{n}\right)-\left(1-p_{1}\right) w_{0}^{n}\right)
$$

\footnotetext{
${ }^{13}$ Under the maintained assumptions we can rule out the possibility that both of the contracts are low-powered.
} 


$$
=p_{1}\left(V-\theta w_{1}^{c}-(1-\theta) w_{1}^{n}\right)-\left(1-p_{1}\right)\left(\theta w_{0}^{c}+(1-\theta) w_{0}^{n}\right) .
$$

Lemma 2 Consider a class of contracts in which both of the contracts are high-powered. Under Assumptions 1 and 2, (i) if $2 \delta \theta(1-\gamma)>(1-\delta)(2 \gamma-1)$, the optimal contract within this class is given by

$$
\mathbf{w}=\left(0, \frac{d}{\Delta p}, 0, \alpha(\gamma, \delta, \theta) \frac{d}{\Delta p}\right)
$$

where

$$
\alpha(\gamma, \delta, \theta) \equiv \frac{2 \gamma(1-\delta+\delta \theta) \Delta p+2 \delta \theta p_{0}}{(1-\delta) \Delta p+2 \delta \theta p_{1}}
$$

(ii) if not, the optimal contract is given by

$$
\mathbf{w}=\left(0, \frac{d}{\Delta p}, 0, \beta(\gamma, \delta, \theta) \frac{d}{\Delta p}\right),
$$

where

$$
\beta(\gamma, \delta, \theta) \equiv \frac{2 \gamma(1-\delta+\delta \theta)}{1-\delta+2 \delta \theta}
$$

Proof: See Appendix.

If $2 \delta \theta(1-\gamma) \leq(1-\delta)(2 \gamma-1), \beta(\gamma, \delta, \theta) \geq 1$ so the type 2 contract is always dominated by the type 1 contract. This indicates that $2 \delta \theta(1-\gamma)>(1-\delta)(2 \gamma-1)$ is the necessary condition for the type 2 contract to be optimal. Note that this necessary condition is satisfied when $\delta$ is sufficiently close to unity.

\section{Type 3: Only the team contract is high-powered}

Finally, we consider a case where only the team contract is high-powered, i.e.,

$$
0.5 \Delta p\left(w_{1}^{c}-w_{0}^{c}\right)+\delta\left(U_{C}-U_{N}\right) \geq \gamma d \quad \text { and } \quad d>\Delta p\left(w_{1}^{n}-w_{0}^{n}\right)
$$

As above, we denote the expected profit per agent in this case by $\pi_{l h}$ where

$$
\pi_{l h}=\theta\left(p_{1}\left(V-w_{1}^{c}\right)-\left(1-p_{1}\right) w_{0}^{c}\right)+(1-\theta)\left(p_{0}\left(V-w_{1}^{n}\right)-\left(1-p_{0}\right) w_{0}^{n}\right) .
$$


Lemma 3 Consider a class of contracts in which only the team contract is high-powered. Under Assumptions 1 and 2, the optimal contract within this class is given by

$$
\mathbf{w}=\left(0,0,0, \beta(\gamma, \delta, \theta) \frac{d}{\Delta p}\right)
$$

Proof: See Appendix.

\subsection{The optimal form of contract in the repeated setting}

With those results we are now ready to determine the optimal form of contract in the repeated setting. Under the maintained assumptions, in the static setting, the optimal individual contract is high-powered while the optimal team contract is low-powered. We now show that this conclusion does not necessarily hold in the repeated setting. To illustrate this point, we confine our attention to a limiting case where $\delta \rightarrow 1$. In this case we can show the following.

Proposition 2 (Collaboration under Weak Complementarities) In the limiting case where $\delta \rightarrow 1$, the optimal team contract is necessarily high-powered.

Proof: See Appendix.

Collaboration is more profitable for the principal if and only if $w_{1}^{c}<w_{1}^{n}$. As $\delta \rightarrow 1$, this condition becomes

$$
\begin{gathered}
\lim _{\delta \rightarrow 1} \alpha(\gamma, \delta, \theta)=\frac{\gamma p_{1}+(1-\gamma) p_{0}}{p_{1}}<1, \\
\lim _{\delta \rightarrow 1} \beta(\gamma, \delta, \theta)=\gamma<1
\end{gathered}
$$

This indicates that in the limiting case, the degree of complementarities needed to make collaboration more profitable can be arbitrarily small: that is, collaboration can be more profitable under arbitrarily weak complementarities. Conversely, though, it is also true that the presence of complementarities is necessary to make collaboration profitable while its magnitude can be arbitrarily small. 
Given this result, the principal is now left with two alternatives, either type 2 or type 3 . Equivalently, the question comes down to whether the optimal individual contract should be high-powered. While the optimal individual contract is high-powered in the static setting by design, we show that this conclusion may be overturned in the repeated setting.

Proposition 3 In the limiting case, there exists some $\bar{\theta}$ such that the optimal individual contract is low-powered for all $\theta \in[\bar{\theta}, 1)$

Proof: See Appendix.

The proposition indicates that there exists a situation where the optimal individual contract is low-powered as long as: (i) the agents are sufficiently patient so that they have vested interests in maintaining their reputation; and (ii) the opportunity to collaborate arrives sufficiently frequently. The result is reasonably intuitive. First, the peer sanction mechanism is more effective when the agents care more about the future. An extreme case is the static setting $(\delta=0)$ where there is no room for the effect of the peer sanction mechanism to take place. Second, the frequency of the opportunity to collaborate also plays an important role on two accounts. On one hand, the more frequently the opportunity arrives, the more the agents lose by defecting and thus destroying a cooperative relationship with the collaborator. The peer sanction mechanism is hence more effective when $\theta$ is sufficiently large. On the other hand, the cost of offering low-powered incentives for non-collaborative work monotonically decreases as the agents can collaborate more frequently. One can then certainly argue that an expansion in the size of the profession facilitates collaboration (an increase in $\theta$ ), which in turn accelerates the tendency to reward lengthy curriculum vitae. ${ }^{14}$

\subsection{Welfare}

We now examine the effect of infinitely repeated interactions on social welfare. In general, one cannot assert whether the optimal dynamic contract promotes efficiency compared to its static counterpart.

\footnotetext{
${ }^{14} \mathrm{As}$ for the increase in the number of collaborative works, Hudson (1996) raises three potential reasons for the recent increase in the number of collaborative works. One of them is the recent growth of the size of the profession.
} 
In either case, an inefficiency may possibly arise but for different reasons.

Under the maintained payoff structure, it is socially efficient to (i) induce effort in all periods, and (ii) engage in collaboration whenever possible (because of complementarities $\gamma<1$ ). First, consider the optimal static contract. Under the optimal static contract, the agents always exert effort, and it is socially efficient in this sense. Under the maintained assumptions, however, the optimal static contract does not encourage collaboration, which entails efficiency losses. The optimal static contract fails to achieve the socially efficient outcome because of the free-rider problem. Since the observable signal is now less sensitive to each agent's behavior, an agent can free-ride on the other agent's effort, and the cost of inducing effort necessarily increases for collaborative work. Under plausible circumstances, this additional cost of inducing effort outweighs the benefit from collaboration: in this case, the principal chooses to let the agents work individually even when it is socially efficient to let them work jointly.

Now consider the optimal dynamic contract. In the repeated setting, the socially efficient outcome is achieved when type (ii) is optimal: in this case, repeated interactions allow the principal to overcome the free-rider problem. When both $\delta$ and $\theta$ are sufficiently close to unity, however, only the team contract is high-powered. Apparently, this type of contract fails to implement the first-best effort when the opportunity to collaborate does not arrive. This inefficiency (the failure to induce effort for non-collaborative works) stems from the principal's incentive to make the peer sanction mechanism more effective, contrary to the case of the optimal static contract where the inefficiency arises from the free-rider problem. This indicates that the current compensation scheme in our profession, which seems to reward collaboration more heavily, may be socially inefficient because it fails to provide proper incentives to induce the first-best effort for non-collaborative works. The following conclusion is immediately obtained from proposition 3 .

Proposition 4 In the limiting case, the optimal contract in the repeated setting is socially efficient if and only if $\theta \in(0, \bar{\theta})$.

Note also that the effect of infinitely repeated interactions on the agents' welfare is ambiguous. To see this, define $U_{h l}, U_{h h}$ and $U_{l h}$ as the agent's expected payoff under the type 1, 2 and 3 contract 
respectively. First, when only the individual contract is high-powered, we obtain

$$
U_{h l}=p_{1} w_{1}^{n}-d=\frac{p_{0} d}{\Delta p} .
$$

This is the agent's expected payoff when $\delta$ is sufficiently small, and hence the expected payoff in the static setting. As $\delta$ approaches unity, either the type 2 or 3 contract emerges as the optimal contract. When both of the contracts are high-powered, the expected payoff is

$$
\begin{aligned}
U_{h h} & =\theta\left(p_{1} w_{1}^{c}-\gamma d\right)+(1-\theta)\left(p_{1} w_{1}^{n}-d\right) \\
& =\theta\left(p_{1} \alpha(\gamma, \delta, \theta) \frac{d}{\Delta p}-\gamma d\right)+(1-\theta) \frac{p_{0} d}{\Delta p} .
\end{aligned}
$$

if $2 \delta \theta(1-\gamma) \leq(1-\delta)(2 \gamma-1)$. We can show that the agents are made better off under the type 2 contract than under the type 1 contract, i.e., $U_{h h} \geq U_{h l}$. This condition can be written as

$$
p_{1} \alpha(\gamma, \delta, \theta) \frac{d}{\Delta p}-\gamma d \geq \frac{p_{0} d}{\Delta p},
$$

which is further simplified to

$$
\alpha(\gamma, \delta, \theta) \geq \frac{\gamma p_{1}+(1-\gamma) p_{0}}{p_{1}} .
$$

It is straightforward to verify that this condition holds when $\gamma>0.5$ as assumed. The expected payoff is monotonically decreasing in $\delta$ and converges to that in the static setting $U_{h l}$ as $\delta \rightarrow 1$.

Finally, when only the team contract is high-powered, the expected payoff in the limiting case is

$$
\begin{aligned}
U_{l h} & =\theta\left(p_{1} w_{1}^{c}-\gamma d\right) \\
& =\theta\left(p_{1} \beta(\gamma, \delta, \theta) \frac{d}{\Delta p}-\gamma d\right) .
\end{aligned}
$$

In this case, the agents may or may not be made better off. When $\delta$ is sufficiently close to unity, however, they are made strictly worse off since $\lim _{\delta \rightarrow 1} \beta(\gamma, \delta, \theta)=\gamma$. In this sense, the agents' desire to maintain reputation may backfire and actually be detrimental to them. The agents suffer in the repeated setting for two reasons. First, they suffer because the principal needs to offer less to induce effort for collaborative work because she can effectively exploit the agents' ability to monitor each other in the repeated setting. Second, they also suffer because they are given high-powered incentives 
only for collaborative works, but there is a positive probability that the opportunity to collaborate does not arrive. We summarize this result as follows.

Proposition 5 In the limiting case, if $\theta \in[\bar{\theta}, 1)$, the agents are made strictly worse off in the repeated setting than in the static setting.

\section{Conclusion}

The paper examines the optimal provision of incentives under voluntary team formation. When collaboration allows the agents to mutually monitor each other, the principal may benefit from encouraging collaboration even in the absence of strong productive complementarities. Moreover, under certain conditions, it is optimal to offer low-powered incentives for non-collaborative works as it makes the peer sanction mechanism more effective. We argue that seemingly low-powered incentives offered for non-collaborative works, as often observed in our profession, can be seen as a critical ingredient of optimal incentive schemes. While our model simply aims at providing an alternative view and is by no means to exclude other potential explanations, we believe that the desire to maintain reputation is critical for academic researchers and the current compensation scheme is a fairly effective way to exploit this desire.

As a final note, there are several unresolved issues that need to be settled. One possibility is to consider the case with multiple principals who independently design compensation schemes. Although the extension does not completely alter the nature of the problem as long as agents are not completely mobile, it certainly makes the problem more complicated since this may allow us to endogenize the probability with which an opportunity to collaborate arrives. A natural way to model this is that the probability is an increasing function of the number of principals offering a high-powered team contract. It is expected that there arises a type of strategic complementarity among the principals because it is certainly not a good idea to offer a low-powered individual contract when others offer a high-powered individual contract and hence not many agents are willing to collaborate. With multiple principals, competition among them also becomes an issue. 
Another potential avenue to extend the current analysis is to allow for more than two agents. For instance, it is more realistic to construct a model where there are many agents and each agent searches for a potential collaborator in a search environment. This type of setup may require some sort of community enforcement, in the sense of Kandori (1992), to make the peer sanction mechanism effective. In this context it naturally becomes crucial how each agent can establish (or tarnish) his reputation or alternatively how the information spreads out in the community when a defection occurs. The peer sanction mechanism is expected to be weak if there are many agents (so that the likelihood of meeting the same agent is negligible) and the information transmission is rather slow. On the other hand, the peer sanction mechanism can be highly effective if each of us belongs to a small community (a guild) from which to choose potential collaborators and the information is immediately shared within the community. In future it is of some interest to pursue this possibility.

\section{Appendix}

Proof of Proposition 1: We first examine the optimal individual contract. It is immediate to see $w_{0}^{n}=0$. It also follows from (3) that the optimal individual contract takes one of the two forms: $w_{1}^{n}=d / \Delta p$, or $w_{1}^{n}=0$. If the principal offers the high-powered contract $w_{1}^{n}=d / \Delta p$, the expected profit is $p_{1}(V-d / \Delta p)$; if she offers the low-powered contract $w_{1}^{n}=0$, it is $p_{0} V$. The principal thus chooses the high-powered contract if

$$
\Delta p V \geq \frac{d}{\Delta p}
$$

Under Assumption 1, therefore, it is optimal for the principal to offer the high-powered individual contract.

We now shift our attention to the optimal team contract and see whether it can improve upon the optimal individual contract. To this end, we first show that the low-powered team contract can never yield more profit than the high-powered individual contract under Assumption 1. Note first that $w_{0}^{c}=0$. Given this, if $w_{1}^{c}=0$, the expected profit is $p_{0} V$. It then follows directly from the argument above that the low-powered team contract cannot improve upon the high-powered individual 
contract. Now consider the high-powered team contract $w_{1}^{c}=\gamma d / \Delta f(1)$. Under this contract, the expected profit is $p_{1}(V-\gamma d / \Delta f(1))$. The team contract improves upon the individual contract if

$$
p_{1}\left(V-\frac{2 \gamma d}{\Delta p}\right) \geq p_{1}\left(V-\frac{d}{\Delta p}\right),
$$

which never holds under Assumption 2. This indicates that the principal should never encourage collaboration: one of the team contracts that can achieve this is $\mathbf{w}^{c}=(0,0)$ while there are certainly many others.

Q.E.D.

Proof of Lemma 2: Before we proceed, we first conjecture that $0.5 \Delta p\left(w_{1}^{c}-w_{0}^{c}\right)<\gamma d$ so that it is stage-game Nash equilibrium to exert no effort for collaborative works, i.e., $u_{N}^{c}=p_{0} w_{1}^{c}+\left(1-p_{0}\right) w_{0}^{c}$. For the high-powered team contract to be meaningful, it is also necessary that the agents choose to collaborate in state $C$ when they can, i.e., $u_{C}^{c} \geq u^{n}$. We suppose for now that these conditions hold and later verify that this is indeed the case. The incentive compatibility constraint for collaborative works is then given by

$0.5 \Delta p\left(w_{1}^{c}-w_{0}^{c}\right)+\frac{\delta \theta}{1-\delta}\left(p_{1} w_{1}^{c}+\left(1-p_{1}\right) w_{0}^{c}-\gamma d-\max \left\{p_{1} w_{1}^{n}+\left(1-p_{1}\right) w_{0}^{n}-d, p_{0} w_{1}^{c}+\left(1-p_{0}\right) w_{0}^{c}\right\}\right) \geq \gamma d$.

Provided that the individual contract is high-powered, it is immediate to see $w_{0}^{n}=0$ and $w_{1}^{n}=d / \Delta p$.

The constraint can be written as

$$
0.5 \Delta p\left(w_{1}^{c}-w_{0}^{c}\right)+\frac{\delta \theta}{1-\delta}\left(p_{1} w_{1}^{c}+\left(1-p_{1}\right) w_{0}^{c}-\gamma d-\max \left\{p_{1} \frac{d}{\Delta p}-d, p_{0} w_{1}^{c}+\left(1-p_{0}\right) w_{0}^{c}\right\}\right) \geq \gamma d .
$$

There are two distinct situations we need to consider: (Case 1) $p_{1} d / \Delta p-d>p_{0} w_{1}^{c}+\left(1-p_{0}\right) w_{0}^{c}$ and (Case 2) $p_{1} d / \Delta p-d \leq p_{0} w_{1}^{c}+\left(1-p_{0}\right) w_{0}^{c}$. We now examine each case in turn.

(Case 1): Suppose that $p_{1} d / \Delta p-d>p_{0} w_{1}^{c}+\left(1-p_{0}\right) w_{0}^{c}$, which means that the agents never collaborate in state $N$. Under this condition, the constraint now becomes

$$
0.5 \Delta p\left(w_{1}^{c}-w_{0}^{c}\right)+\frac{\delta \theta}{1-\delta}\left(p_{1} w_{1}^{c}+\left(1-p_{1}\right) w_{0}^{c}-\gamma d-p_{1} \frac{d}{\Delta p}+d\right) \geq \gamma d .
$$


We first show $w_{0}^{c}=0$. To show this, suppose that the optimal team contract is given by $\mathbf{w}^{c}=(a, b)$ where $a>0$. Note, however, that another contract $\mathbf{w}^{c}=\left(0,\left(p_{1} b+\left(1-p_{1}\right) a\right) / p_{1}\right)$ yields the same profit level and satisfies the constraint with strict inequality. This is a contradiction and we can thus conclude $w_{0}^{c}=0$. Given this result, the constraint can be simplified to

$$
0.5 \Delta p w_{1}^{c}+\frac{\delta \theta}{1-\delta}\left(p_{1} w_{1}^{c}-\gamma d-p_{1} \frac{d}{\Delta p}+d\right) \geq \gamma d
$$

Solving this constraint, we obtain a candidate team contract

$$
w_{0}^{c}=0, w_{1}^{c}=\frac{2 \gamma(1-\delta+\delta \theta) \Delta p+2 \delta \theta p_{0}}{(1-\delta) \Delta p^{2}+2 \delta \theta p_{1} \Delta p} d .
$$

To show that this contract implements the desired outcome, it is necessary that (i) $\gamma d>0.5 \Delta p w_{1}^{c}$ and (ii) $u_{C}^{c} \geq u^{n}$, i.e., $p_{1} w_{1}^{c}-\gamma d \geq p_{1} w_{1}^{n}$. It is straightforward to see that the first condition is always satisfied when $\gamma>0.5$. The second condition can be written as

$$
p_{1} \frac{2 \gamma(1-\delta+\delta \theta) \Delta p+2 \delta \theta p_{0}}{(1-\delta) \Delta p^{2}+2 \delta \theta p_{1} \Delta p} d-\gamma d \geq p_{1} \frac{d}{\Delta p}-d=\frac{p_{0} d}{\Delta p}
$$

which is reduced to

$$
p_{1} \frac{2 \gamma(1-\delta+\delta \theta) \Delta p+2 \delta \theta p_{0}}{(1-\delta) \Delta p+2 \delta \theta p_{1}} \geq \gamma p_{1}+(1-\gamma) p_{0}
$$

With some algebra, one can show that this condition is always satisfied when $\gamma>0.5$.

The optimal team contract must also satisfy $p_{1} d / \Delta p-d>p_{0} w_{1}^{c}$, which can also be written as $d / \Delta p>w_{1}^{c}$. This holds if

$$
1>\frac{2 \gamma(1-\delta+\delta \theta) \Delta p+2 \delta \theta p_{0}}{(1-\delta) \Delta p+2 \delta \theta p_{1}},
$$

which is further simplified to

$$
2 \delta \theta(1-\gamma)>(1-\delta)(2 \gamma-1)
$$

Finally, we need to show that the candidate contract is collusion-proof, i.e.,

$$
\Delta p w_{1}^{c} \geq \gamma d
$$

This condition can be written as

$$
\frac{2 \gamma(1-\delta+\delta \theta) \Delta p+2 \delta \theta p_{0}}{(1-\delta) \Delta p+2 \delta \theta p_{1}} \geq \gamma
$$


With some algebra, one can show that this condition always holds. This implies that if (34) is satisfied, the optimal team contract within this class is given by (30).

(Case 2): Now suppose that $p_{1} d / \Delta p-d \leq p_{0} w_{1}^{c}+\left(1-p_{0}\right) w_{0}^{c}$, which means that the agents choose to collaborate even in state $N$. Provided that $0.5 \Delta p\left(w_{1}^{c}-w_{0}^{c}\right)<\gamma d$, the incentive compatibility constraint for collaborative works becomes

$$
0.5 \Delta p\left(w_{1}^{c}-w_{0}^{c}\right)+\frac{\delta \theta}{1-\delta}\left(p_{1} w_{1}^{c}+\left(1-p_{1}\right) w_{0}^{c}-\gamma d-p_{0} w_{1}^{c}-\left(1-p_{0}\right) w_{0}^{c}\right) \geq \gamma d
$$

This condition can further be written as

$$
0.5 \Delta p\left(w_{1}^{c}-w_{0}^{c}\right)+\frac{\delta \theta}{1-\delta}\left(\Delta p\left(w_{1}^{c}-w_{0}^{c}\right)-\gamma d\right) \geq \gamma d
$$

Solving this we obtain

$$
w_{1}^{c}-w_{0}^{c} \geq \frac{2 \gamma(1-\delta+\delta \theta) d}{(1-\delta+2 \delta \theta) \Delta p}
$$

which implies $w_{0}^{c}=0$.

To show that this contract implements the desired outcome, it is necessary that (i) $\gamma d>0.5 \Delta p w_{1}^{c}$ and (ii) $u_{C}^{c} \geq u^{n}$, i.e., $p_{1} w_{1}^{c}-\gamma d \geq p_{1} w_{1}^{n}-d$. It is immediate to see that the first condition is always satisfied. Note also that

$$
\frac{2 \gamma(1-\delta+\delta \theta)}{1-\delta+2 \delta \theta} \geq 1
$$

when $2 \delta \theta(1-\gamma) \leq(1-\delta)(2 \gamma-1)$. This implies that the second condition is also satisfied.

The optimal team contract must also satisfy $p_{1} d / \Delta p-d \leq p_{0} w_{1}^{c}+\left(1-p_{0}\right) w_{0}^{c}$, which can be simplified to

$$
w_{1}^{c} \geq \frac{d}{\Delta p}
$$

Note that this condition always holds when $2 \delta \theta(1-\gamma) \leq(1-\delta)(2 \gamma-1)$. It follows from this that the candidate contract is given by

$$
w_{0}^{c}=0, w_{1}^{c}=\frac{2 \gamma(1-\delta+\delta \theta) d}{(1-\delta+2 \delta \theta) \Delta p},
$$


if $2 \delta \theta(1-\gamma) \leq(1-\delta)(2 \gamma-1)$.

Finally, it directly follows from (41) that this candidate contract is also collusion-proof, i.e., $\Delta p w_{1}^{c} \geq$ $\gamma d$. This implies that if (34) is not satisfied, the optimal team contract within this class is given by (42).

Q.E.D.

Proof of Lemma 3: As above, we first conjecture (i) $0.5 \Delta p\left(w_{1}^{c}-w_{0}^{c}\right)<\gamma d$ and (ii) $u_{C}^{c} \geq u^{n}$. Given these, the incentive compatibility constraint for collaborative works becomes

$$
0.5 \Delta p\left(w_{1}^{c}-w_{0}^{c}\right)+\frac{\delta \theta}{1-\delta}\left(p_{1} w_{1}^{c}+\left(1-p_{1}\right) w_{0}^{c}-\gamma d-\max \left\{p_{0} w_{1}^{n}+\left(1-p_{0}\right) w_{0}^{n}, p_{0} w_{1}^{c}+\left(1-p_{0}\right) w_{0}^{c}\right\}\right) \geq \gamma d
$$

If the individual contract is low-powered, there is no reason to offer a positive wage for noncollaborative works, implying $w_{0}^{n}=w_{1}^{n}=0$ and $u^{n}=0$. The incentive compatibility constraint then becomes

$$
0.5 \Delta p\left(w_{1}^{c}-w_{0}^{c}\right)+\frac{\delta \theta}{1-\delta}\left(\Delta p\left(w_{1}^{c}-w_{0}^{c}\right)-\gamma d\right) \geq \gamma d
$$

which leads to

$$
w_{1}^{c}-w_{0}^{c} \geq \frac{2(1-\delta+\delta \theta) \gamma d}{(1-\delta+2 \delta \theta) \Delta p} .
$$

The candidate contract is thus given by

$$
w_{0}^{c}=0, w_{1}^{c}=\frac{2(1-\delta+\delta \theta) \gamma d}{(1-\delta+2 \delta \theta) \Delta p},
$$

which indeed satisfies $\gamma d>0.5 \Delta p w_{1}^{c}$ for any $(\delta, \theta) \in(0,1)^{2}$ as conjectured. It is also immediate to see that $u_{C}^{c} \geq u^{n}=0$.

Finally, we need to verify that the candidate contract is collusion-proof, i.e.,

$$
\Delta p w_{1}^{c}=\frac{2(1-\delta+\delta \theta) \gamma d}{1-\delta+2 \delta \theta} \geq \gamma d
$$

which can be simplified to

$$
2(1-\delta+\delta \theta) \geq 1-\delta+2 \delta \theta
$$

Since this evidently holds, the optimal team contract within this class is given by (46). 
Q.E.D.

Proof of Proposition 2: To prove the proposition it suffices to show $\pi_{h h}>\pi_{h l}$ when $\delta$ is arbitrarily close to unity. It follows from lemma 2 that as $\delta \rightarrow 1$, the optimal contract is $(0, d / \Delta p, 0, \alpha(\gamma, \delta, \theta) d)$ when both of the contracts are high-powered. Note that

$$
\lim _{\delta \rightarrow 1} \alpha(\gamma, \delta, \theta)=\frac{\gamma \Delta p+p_{0}}{p_{1}} .
$$

This implies that as $\delta \rightarrow 1$,

$$
\pi_{h h}=p_{1}\left(V-\theta \frac{\gamma \Delta p+p_{0}}{p_{1} \Delta p} d-(1-\theta) \frac{d}{\Delta p}\right) .
$$

Note also that the expected profit under the type 1 contract is independent of $\delta$ :

$$
\pi_{h l}=p_{1}\left(V-\frac{d}{\Delta p}\right)
$$

Given $p_{0}>0$, it is immediate to see $\pi_{h h}>\pi_{h l}$ as $\delta \rightarrow 1$.

Q.E.D.

Proof of Proposition 3: The optimal individual contract is low-powered if $\pi_{l h} \geq \pi_{h h}$. Notice that

$$
\lim _{\delta \rightarrow 1} \beta(\gamma, \delta, \theta)=\gamma
$$

As $\delta \rightarrow 1, \pi_{l h} \geq \pi_{h h}$ if

$$
\theta p_{1}\left(V-\frac{\gamma d}{\Delta p}\right) \geq p_{1}\left(V-\theta \frac{\gamma \Delta p+p_{0}}{p_{1} \Delta p} d-(1-\theta) \frac{d}{\Delta p}\right),
$$

which is reduced to

$$
\frac{\theta(1-\gamma) p_{0} d+(1-\theta) p_{1} d}{p_{1} \Delta p} \geq(1-\theta) V .
$$

It is easy to see that this condition holds as $\theta \rightarrow 1$.

Q.E.D. 


\section{References}

Baker, George; Gibbons, Robert and Murphy, Kevin J. "Subjective Performance Measures in Optimal Incentive Contracts." Quarterly Journal of Economics, November 1994, 109(4), pp. 1125-56.

. "Relational Contracts and the Theory of the Firm." Quarterly Journal of Economics, February 2002, 117(1), pp. 39-84.

Bernheim, B. Douglas and Whinston, Michael D. "Incomplete Contracts and Strategic Ambiguity." American Economic Review, September 1998, 88(4), pp. 902-32.

Bull, Clive. "The Existence of Self-Enforcing Implicit Contracts." Quarterly Journal of Economics, February 1987, 102(1), pp. 147-59.

Che, Yeon-Koo and Yoo, Seung-Weon. "Optimal Incentives for Teams." American Economic Review, June 2001, 91(3), pp. 525-41.

Durden, Garey C. and Perri, Timothy J. "Coauthorship and Publication Efficiency." Atlantic Economic Journal, March 1995, 23(1), pp. 69-76.

Engers, Maxim; Gans, Joshua S.; Grant, Simon and King, Stephen P. "First-Author Conditions." Journal of Political Economy, August 1999, 107(4), pp. 859-83.

Heck, Jean L. and Zaleski, Peter A. "Trends in Economic Journal Literature: 1969-89." Atlantic Economic Journal, December 1991, 19(4), pp. 27-32.

Hollis, Aidan. "Coauthorship and the Output of Academic Economists." Labour Economics, September 2001, 8(4), pp. 503-30.

Hudson, John. "Trends in Multi-Authored Papers in Economics." Journal of Economic Perspectives, Summer 1996, 10(3), pp. 153-8.

Ishida, Junichiro. "Team Incentives under Relative Performance Evaluation." forthcoming in Journal of Economics and Management Strategy, 2004. 
Kandori, Michihiro. "Social Norms and Community Enforcement." Review of Economic Studies, January 1992, 59(1), pp. 63-80.

Laband, David N. and Tollison, Robert D. "Intellectual Collaboration." Journal of Political Economy, June 2000, 108(3), pp. 632-62.

Levin, Jonathan. "Relational Incentive Contracts." American Economic Review, June 2003, 93(3), pp. $835-57$.

MacLeod, W. Bentley. "Optimal Contracting with Subjective Evaluation." American Economic Review, March 2003, 93(1), pp. 216-40.

MacLeod, W. Bentley and Malcomson, James M. "Implicit Contracts, Incentive Compatibility, and Involuntary Unemployment." Econometrica, March 1989, 57(2), pp. 447-80.

McDowell, John M. and Smith, Janet K. "The Effect of Gender-Sorting on Propensity to Coauthor: Implications for Academic Promotion." Economic Inquiry, January 1992, 30(1), pp. 68-82.

Rayo, Luis. "Relational Team Incentives and Ownership." mimeo, 2003.

Sauer, Raymond D. "Estimates of the Returns to Quality and Coauthorship in Economic Academics." Journal of Political Economy, August 1988, 96(4), pp. 855-66. 